\title{
Lipid Reconstitution-Enabled Formation of Gold Nanoparticle Clusters for Mimetic Cellular Membrane
}

\author{
Jiyoung Nam, ${ }^{1}$ Yong-Tae Kim, ${ }^{1}$ Aeyeon Kang, ${ }^{2}$ Kook-Han Kim, ${ }^{1}$ KyoRee Lee, ${ }^{3}$ \\ Wan Soo Yun, ${ }^{2}$ and Yong Ho Kim ${ }^{1,2}$ \\ ${ }^{1}$ SKKU Advanced Institute of Nanotechnology, Sungkyunkwan University, Suwon 16419, Republic of Korea \\ ${ }^{2}$ Department of Chemistry, Sungkyunkwan University, Suwon 16419, Republic of Korea \\ ${ }^{3}$ Interdisciplinary Course of Physics and Chemistry, Sungkyunkwan University, Suwon 16419, Republic of Korea \\ Correspondence should be addressed to Wan Soo Yun; wsyun87@skku.edu and Yong Ho Kim; yhkim94@skku.edu
}

Received 2 March 2016; Accepted 28 April 2016

Academic Editor: Yaling Liu

Copyright (C) 2016 Jiyoung Nam et al. This is an open access article distributed under the Creative Commons Attribution License, which permits unrestricted use, distribution, and reproduction in any medium, provided the original work is properly cited.

Gold nanoparticles (AuNPs) encapsulated within reconstituted phospholipid bilayers have been utilized in various bioapplications due to their improved cellular uptake without compromising their advantages. Studies have proved that clustering AuNPs can enhance the efficacy of theranostic effects, but controllable aggregation or oligomerization of AuNPs within lipid membranes is still challenging. Here, we successfully demonstrate the formation of gold nanoparticle clusters (AuCLs), supported by reconstituted phospholipid bilayers with appropriate sizes for facilitating cellular uptake. Modulation of the lipid membrane curvatures influences not only the stability of the oligomeric state of the AuCLs, but also the rate of cellular uptake. Dynamic light scattering (DLS) data showed that 1-palmitoyl-2-oleoyl-sn-glycero-3-phosphoethanolamine (POPE), with its relatively small head group, is crucial for establishing an effective membrane curvature to encapsulate the AuCLs. The construction of phospholipid bilayers surrounding AuCLs was confirmed by analyzing the secondary structure of M2 proteins incorporated in the lipid membrane surrounding the AuCLs. When AuCLs were incubated with cells, accumulated clusters were found inside the cells without the lipids being removed or exchanged with the cellular membrane. We expect that our approach of clustering gold nanoparticles within lipid membranes can be further developed to design a versatile nanoplatform.

\section{Introduction}

Gold nanoparticles (AuNPs) have been widely studied in the field of biomedicine for a number of years because of their advantages in biocompatibility and distinguished photothermal properties [1-5]. Recent progress in the synthesis of AuNPs has allowed for precise control of their optical properties, which has motivated the development of multifunctional theranostic nanocomplexes [6-8]. Several studies have shown that the plasmonic effect of nanoparticles is intensified when the distance between particles is reduced, and thereby the surface-enhanced Raman scattering (SERS) signal is significantly amplified within the interparticle region [9-11]. However, the fine-tuning of surface association with functionality and aggregation control of gold nanoparticles toward developing the ultrasensitive biosensors or noninvasive bimodal detection of target diseases are still daunting challenges [11, 12]. Inspired by biological membranes, solidsupported lipid assembly has been widely investigated to provide artificial lipid layers for studying membrane-associated biological phenomena $[13,14]$. Unlike synthetic micelle or liposomes, lipids reconstituted on solids possess structural stability that enables prolonged experiments even for in vivo studies [15]. Liposomes prepared from phospholipids and phospholipid mimics have also been used to deposit various nanoparticles, to enhance biocompatibility and delivery efficiency [16, 17]. For instance, phospholipid-encapsulated quantum dots were used for in vitro and in vivo imaging with reduced toxicity [18], and mesoporous silica nanoparticles within phospholipid envelope were used as drug cargo for cancer treatment [19]. Phospholipid-encapsulated AuNPs have been highlighted as theranostic agents because their lipid coating can provide a platform to design the nanoparticle to target certain molecules within cells while retaining 
the benefits of gold nanoparticles [20-22]. Several methods have been proposed to cluster lipid-coated AuNPs to enhance their therapeutic effect, but the addition of aggregation inducers or chemical modification of lipids was necessary to achieve this aggregation.

In this work, we demonstrate the construction of gold nanoparticle clusters (AuCLs) solely driven by the self-assembly of a synthetic phospholipid membrane, which can possibly be used for multifunctional drug cargo through cellular internalization. The synthesis of AuCLs was followed by two steps: (1) three different kinds of synthetic phospholipids, 1palmitoyl-2-oleoyl-sn-glycero-3-phosphocholine (POPC), 1palmitoyl-2-oleoyl-sn-glycero-3-phospho-L-serine (POPS), and 1-palmitoyl-2-oleoyl-sn-glycero-3-phosphoethanolamine (POPE), were mixed in a molar ratio of $7: 1: 2$, respectively, to create uniformly sized small unilamellar vesicles (SUV); and (2) incubating SUV with $20 \mathrm{~nm}$ AuNPs allowed the reconstitution of phospholipids on the surface of the particles that drive the formation of AuCLs. The presence of lipid layers was confirmed with transmission electron microscopy (TEM), UV-vis spectrometer, and dynamic light scattering (DLS). We analyzed the secondary structure of the M2 protein, a membrane protein from the influenza A virus, to verify the presence of the lipid layer, and this result confirmed that the phospholipids are assembled in bilayers on the surfaces of AuCLs. Furthermore, when cells were treated with AuCLs, hundreds of particles found inside the cells proved the ability of cellular uptake. Overall, this work demonstrates a facile method to cluster nanoparticles without chemical modification and the possibility of developing nanocarriers of various sizes that can be used as nanobiomedicine.

\section{Materials and Methods}

2.1. Materials. 1-Palmitoyl-2-oleoyl-sn-glycero-3-phosphocholine (POPC), 1-palmitoyl-2-oleoyl-sn-glycero-3-phosphoL-serine (sodium salt) (POPS), 1-palmitoyl-2-oleoyl-sn-glycero-3-phosphoethanolamine (POPE), and L- $\alpha$-phosphatidylethanolamine-N-(lissamine rhodamine $\mathrm{B}$ sulfonyl) were obtained from Avanti Polar Lipids, Inc. (Alabaster, AL, USA). A solution of $20 \mathrm{~nm}$ gold nanoparticles $\left(6 \times 10^{12}\right.$ particles per milliliter, stabilized suspension in citrate buffer) was purchased from Sigma-Aldrich (Steinheim, Germany). All solutions were prepared using ultrapure water, obtained using a Millipore Milli-Q water purification system (Darmstadt, Germany).

2.2. Synthesis of Phospholipid-Coated Gold Nanoparticles. For the preparation of liposomes, lipids of POPC, POPS, and POPE with the molar ratio of $7: 1: 2$ were prepared in chloroform and transferred into a small glass vial. Chloroform was slowly evaporated with a gentle stream of nitrogen gas in order to form a thin film of lipid multilayers on the bottom of the glass vial. After lyophilizing for 2 days, distilled water was slowly added until it reached the desired volume, and the solution was sonicated in order to produce homogeneous uniformly sized small unilamellar vesicles (SUV). The final concentration of lipids was $5 \mathrm{mM}$. The solution was centrifuged at $9300 \mathrm{rcf}$ for $10 \mathrm{~min}$, and the supernatant, kept in ice, was used for further experiments.
Phospholipid-coated gold nanoparticles were prepared by mixing a solution of gold nanoparticles and SUV in a volume ratio of $1: 9$ and incubating the mixture at $4^{\circ} \mathrm{C}$ for 12 hours. In order to thoroughly mix the two components, an inverting motion was applied with a rotating mixer. To isolate AuCLs from the mixture of single gold nanoparticles and SUV, the sample solution was centrifuged at 12,000 rpm for $10 \mathrm{~min}$, and the supernatant was removed. The precipitant was resuspended in a $1 \mathrm{x}$ PBS solution. The concentration of gold nanoparticles in AuCLs was measured by UV-vis spectrometer at an absorbance at $525 \mathrm{~nm}$ (UV-1800, Shimadzu, Columbia, MD, USA).

2.3. Particle Characterization. Transmission electron microscopy (TEM) (JEM ATM 200F, JEOL, $200 \mathrm{kV}$ ) and UVvis spectrometer was used to characterize the formation of AuCLs. The size distribution of AuCLs, according to time, was measured by dynamic light scattering (DLS) (ZEN3690, Nano-ZS90, Malvern, UK).

2.4. Synthesis of M2 Protein by Solid-Phase Synthesis. Wildtype M2 protein, spanning the transmembrane helical motif, was synthesized by solid-phase synthesis adopting Fmoc chemistry on the H-Rink amide ChemMatrix ${ }^{\circledR}$ resin $(0.47 \mathrm{mmol} / \mathrm{g}$ loading, PCAS BioMatrix Inc., Quebec, Canada) [23]. The amino acid sequence was SSDPLVVAASIIGILHLILWILDRL. The product was cleaved from the resin with a cleavage cocktail solution containing $95: 2.5: 2.5$ of trifluoroacetic acid (TFA) : triisopropylsilane (TIS): deionized water, in a volume ratio, for 2 hours. Resin was removed by filtration, and the remaining solution was vaporized under nitrogen gas. Purification by reverse phase high-performance liquid chromatography (HPLC, Quaternary Gradient Module 2545, Waters) using a Vydac C4 column employed a linear gradient of buffer $\mathrm{A}\left(99.9 \% \mathrm{H}_{2} \mathrm{O}\right.$ and $0.1 \%$ TFA) and buffer $\mathrm{B}\left(90 \%\right.$ acetonitrile, $9.9 \% \mathrm{H}_{2} \mathrm{O}$, and $0.1 \%$ TFA). The molecular mass of M2 was confirmed by matrix-assisted laser desorption/ionization-time of flight (MALDI-TOF) mass spectroscopy (Ultraflex III, Bruker, Massachusetts, MA, USA).

2.5. Circular Dichroism (CD) Spectroscopy. To analyze the secondary structure of the M2 proton channel, the stock solution of M2 protein was prepared in a $10 \%(\mathrm{w} / \mathrm{v})$ methanol solution. For lipid insertion, $200 \mu \mathrm{M}$ of M2 protein stock solution was added to $30 \mathrm{nM}$ of AuCLs in 1x PBS and incubated for $15 \mathrm{~min}$ with an inverting motion. Excess M2 protein was removed by centrifugation, and the precipitant was resuspended in 1x PBS for analysis. The secondary structure of the M2 proton channel was speculated by CD spectrometer (J1500, Jasco Inc., Maryland, MD, USA). The CD spectra measurement was performed in quartz cells with $1 \mathrm{~mm}$ pathlengths, with the far UV light ranged from $190 \mathrm{~nm}$ to $260 \mathrm{~nm}$.

2.6. Cellular Uptake of AuCLs (Fluorescence). Fluorescentlabeled AuCLs were prepared for optical microscopy. The lipid layer was tagged with 2 mol\% of L- $\alpha$-phosphatidylethanolamine-N-(lissamine rhodamine $B$ sulfonyl), which 


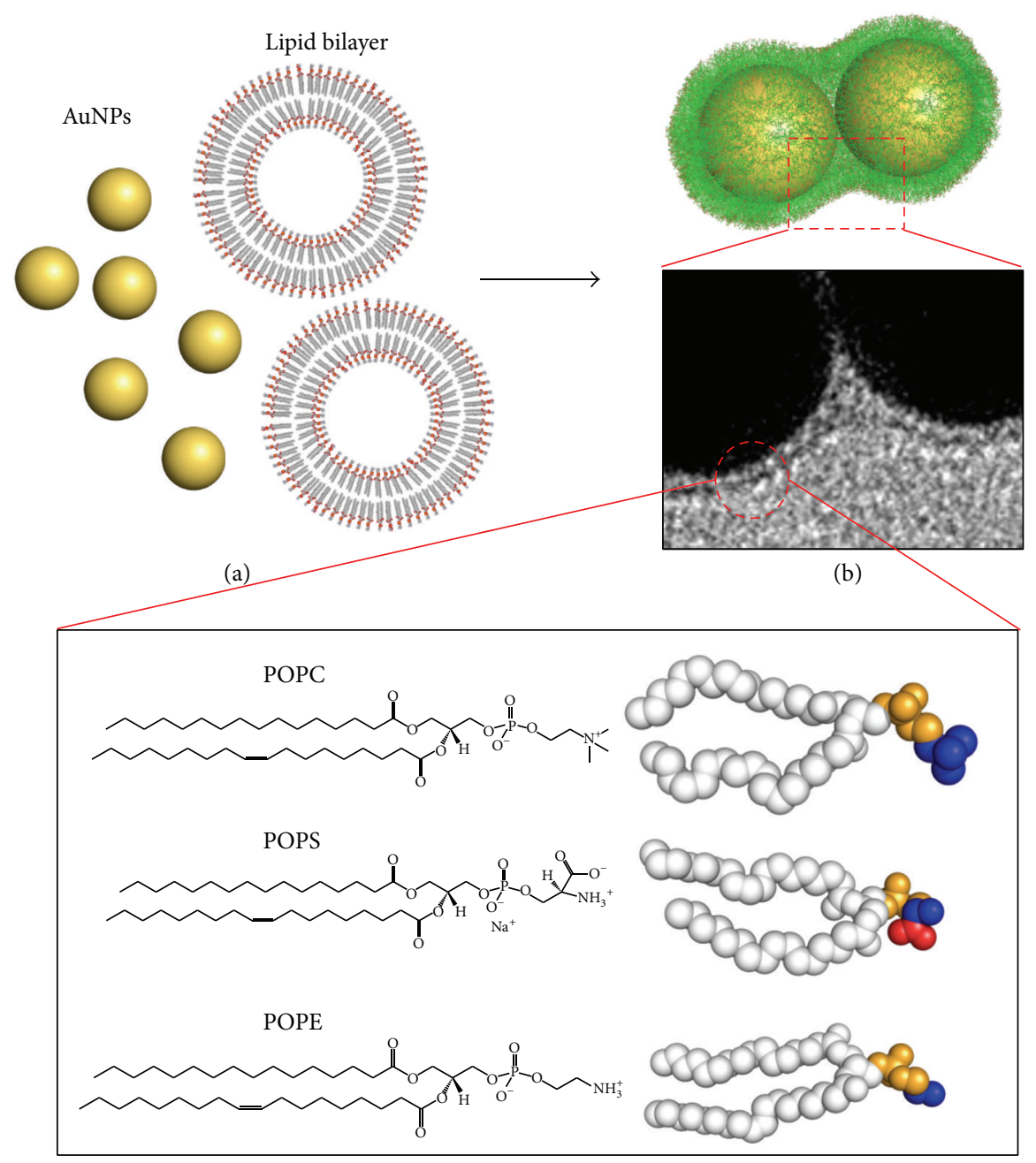

(c)

FIGURE 1: Self-assembling gold nanoparticles with synthetic phospholipid membranes driving gold nanoparticle clustering. (a) Schematic image of the formation of lipid-coated AuCLs. (b) Reconstituted lipid membrane enveloping the surface of AuCLs is visualized in a transmission electron microscopy (TEM) image, which shows surface coverage of a phospholipid membrane surrounding the AuCL dimer with a thickness of $4 \sim 6 \mathrm{~nm}$. Incubation of AuNPs with SUV allows encapsulation of AuCLs. (c) Types of phospholipids used in this study: molecular structures of POPC, POPS, and POPE.

achieved excitation and emission at $560 \mathrm{~nm}$ and $579 \mathrm{~nm}$, respectively.

SH-SY5Y neuroblastoma cells were cultured in Dulbecco's modified Eagle's medium (DMEM) with 10\% fetal bovine serum and incubated at $37^{\circ} \mathrm{C}$ with $5 \% \mathrm{CO}_{2}$. For cellular uptake studies, $2 \times 10^{4} \mathrm{SH}$-SY5Y cells were seeded in 96-well plates, and $5 \mathrm{nM}$ of fluorescence-tagged AuCLs were added in $100 \mu \mathrm{L}$ cell media. After 1 hour of incubation, cells were washed with fresh media and placed on the stand of the microscope (TCS SP8, Leica Microsystems, Germany) for imaging.

\section{Results and Discussion}

3.1. Preparation and Characterization of AuCLs. The AuCLs, illustrated in Figure 1, were prepared by reconstituting phospholipids on the surface of gold nanoparticles. Phospholipids are the most abundant components in cellular membranes and consist of two parts: a hydrophilic head group and hydrophobic alkyl chains [24]. The amphiphilic property of phospholipids leads the molecules to self-assemble into organized structures and the size and shape of the vesicles are highly dependent on the size of head groups and length of side chains $[25,26]$. We utilized three different phospholipid components, including POPC, POPS, and POPE, in a molar ratio of $7: 1: 2$, for formulating uniformly sized SUV. Upon the incubation of citrate-capped AuNPs with SUV, citrates were exchanged with phospholipids and a thin layer of phospholipid membrane was reconstructed on AuNPs.

To confirm the formation of clusters, TEM images of AuCLs were taken (Figure 2(a)). The nanoparticles appeared to be clustered as dimers, trimers, and tetramers, and a few were aggregated in higher numbers. Some particles were found to exist as monomers, but we assume that 

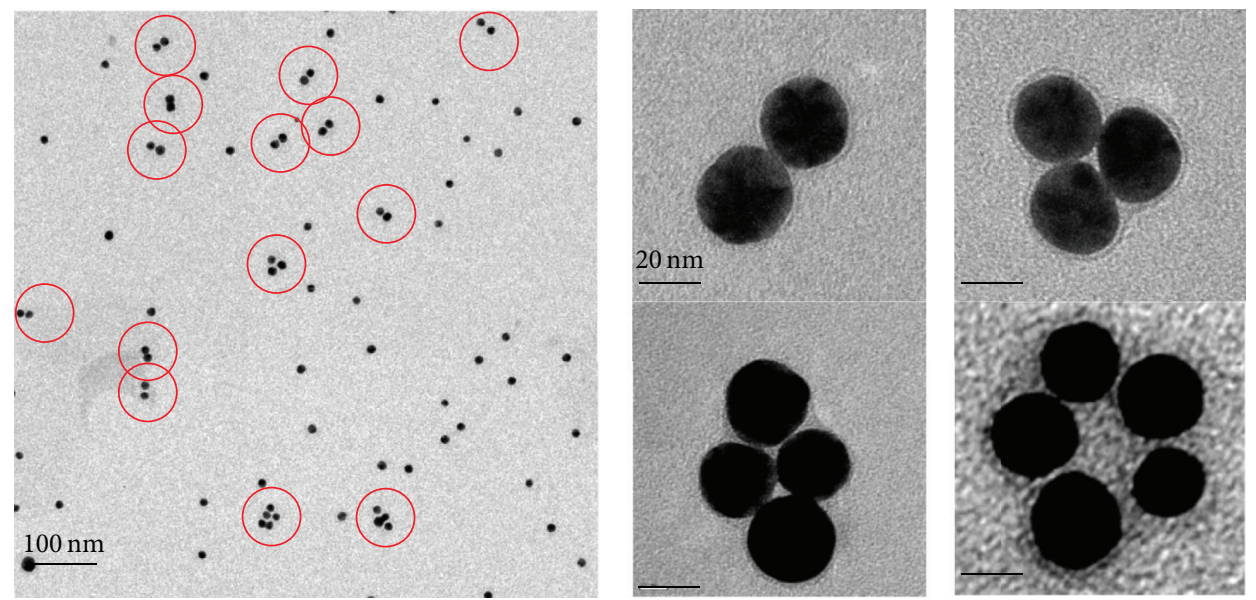

(a)

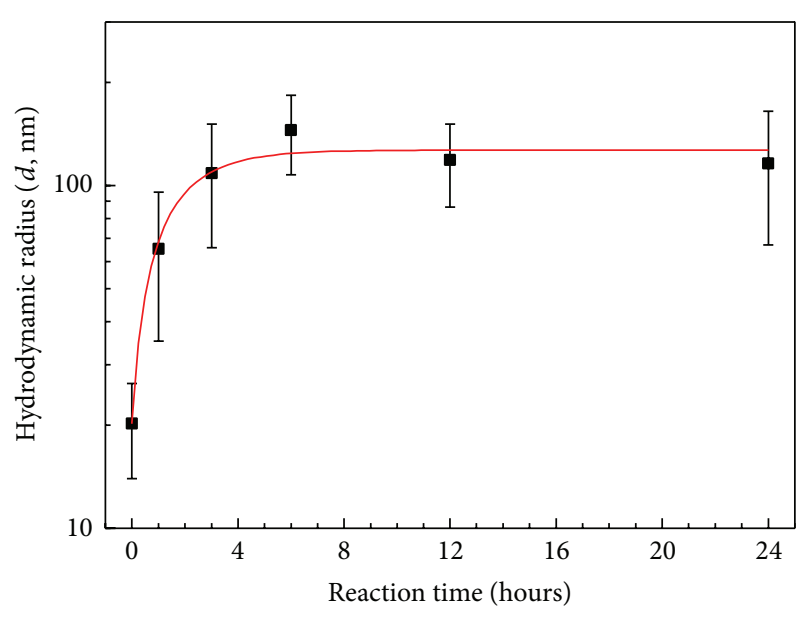

(b)

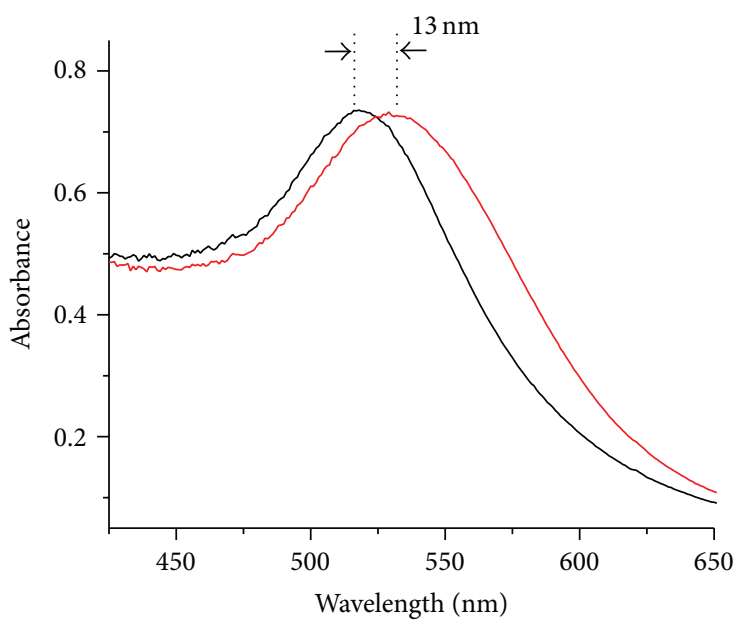

(c)

FIgure 2: Characterization of synthesized AuCLs. (a) TEM image of AuCLs, in low resolution (left) and high resolution (right). Oligomeric states of AuCLs were indicated by red circles. High-resolution TEM images provide direct evidence for the presence of lipid layers enveloping nanoparticle clusters. (b) DLS data for AuCLs as a function of time. Hydrodynamic radius of AuCLs increases until the average size reaches $100 \mathrm{~nm}$ and is sustained for at least 24 hours. (c) UV-vis spectra of AuNPs (black) and AuCLs (red).

the individual particles would have clustered as well if more incubation time had been allowed. High-resolution TEM images at low accelerating voltage in an electron beam, to increase the contrast of lipid compartments, provided direct evidence of the presence of lipid layers surrounding nanoparticle clusters. DLS was used to observe kinetic phenomena of the clustering process and to examine the stability of AuCLs (Figure 2(b)). The hydrodynamic radius of AuCLs was significantly increased for 4 hours and saturated when the average size reached $100 \mathrm{~nm}$. The average size of clusters was sustained for 24 hours, indicating that the lipid layer inhibits uncontrolled aggregation of AuNPs. UV-vis spectra revealed that phospholipid-supported AuCLs caused a slight red shift in a maximum absorbance peak, because of plasmonic coupling in the interparticular regions of AuCLs (Figure 2(c)) [27].

The stiffness and curvature of membranes are highly dependent on their lipid compositions, because lipids having different shapes or charges influence the interaction between each component $[26,28]$. To validate the effect of lipid composition on clustering nanoparticles, lipid vesicles comprising two types of phospholipids, POPC and POPS, were prepared and incubated with nanoparticles. As Figure 3 shows, liposomes made up of POPC, POPS, and POPE clustered nanoparticles well, while the liposomes prepared without POPE failed to generate oligomeric states of nanoparticles. It is assumed that the relatively small head group of POPE stabilizes the stiff lipid curvature on the surfaces of AuCLs and is thus likely to generate tighter membranes on the clusters.

\subsection{Incorporation of M2 Proton Channel and Circular Dichro-} ism (CD) Spectroscopy for Its Secondary Structure Analysis. To verify the formation of lipid bilayers, M2 proteins were incorporated into the AuCLs and the secondary structure of the protein was analyzed (Figure 4). M2 protein is one of the viral proton channels in the membrane of the influenza A virus [23]. It is known to have a tetrameric coiled-coil structure in 


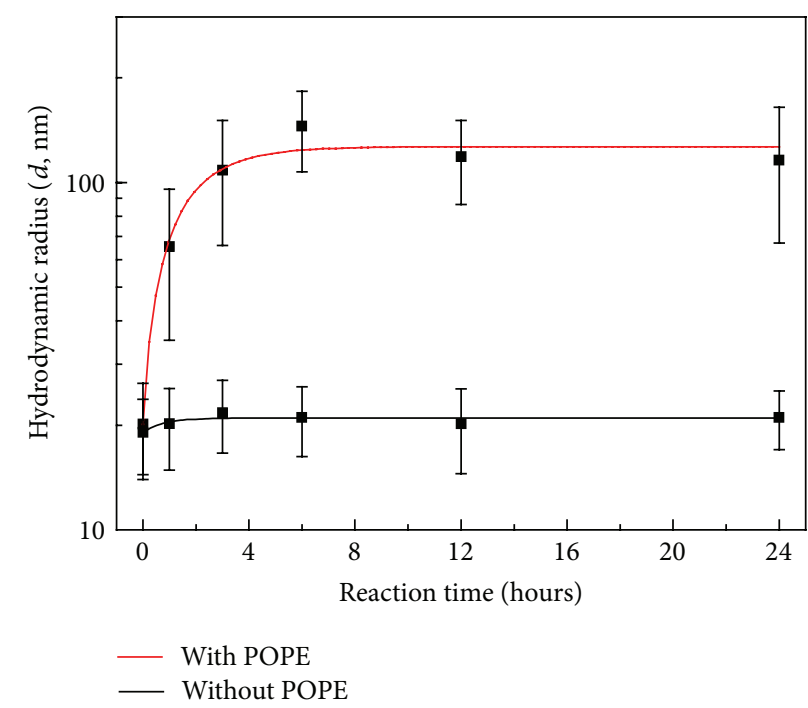

FIgure 3: Hydrodynamic radius of AuCLs with POPC, POPS, and POPE in a molar ratio of $7: 1: 2$ (red) or POPC and POPS in a molar ratio of $9: 1$, respectively (black). The result indicates that POPE is necessary to construct stable lipid curvature on nanoparticles.

natural lipid bilayer environments, while having random coiling in nonmembrane environments $[29,30]$. We anticipated that M2 proteins would exhibit their inherent $\alpha$-helical conformations when incorporated into the lipid layer of AuCLs and would ultimately prove that the phospholipids are assembled into a bilayer (Figure 4(a)). The CD spectra of M2 proteins incubated with AuCLs exhibited strong negative absorption at wavelengths of $208 \mathrm{~nm}$ and $222 \mathrm{~nm}$, consistent with the spectrum of a typical $\alpha$-helical conformation (Figure 4(b)). In order to eliminate the absorbance by the excess M2 protein in solution, unbounded M2 proteins were separated from the AuCLs by centrifugation with a PBS solution. Although the intensity of the peaks decreased, the CD spectrum of the resuspended AuCLs clearly showed the same $\alpha$-helical propensity of fully folded $\alpha$-helices of M2 protein in the literature. The result confirmed that the reconstituted lipid membrane on AuCLs provides a folding environment for M2 proteins by acting as a mimetic of cellular membranes. For control experiments, M2 proteins were incubated with citratecapped monodispersed AuNPs as a negative control. The mixture did not generate any appreciable absorbance peak in the wavelength range from $190 \mathrm{~nm}$ to $260 \mathrm{~nm}$ after same centrifugation process was applied. Overall, these results provide the possibility of expanding the use of AuCLs into therapeutic purposes by conjugating the lipid surface with functional molecules, or targeting cell membrane proteins.

3.3. Cellular Uptake of AuCLs. We next investigated cellular internalization of AuCLs. Fluorescence-tagged POPE was used to visualize AuCLs, and their average size was not changed by the fluorescence-tagging. When the fluorescencetagged AuCLs were incubated with cells, large amount of nanoparticles was found to be internalized within a short period (Figure 5). In spite of crossing biological membrane environments, phospholipids surrounding AuCLs were not

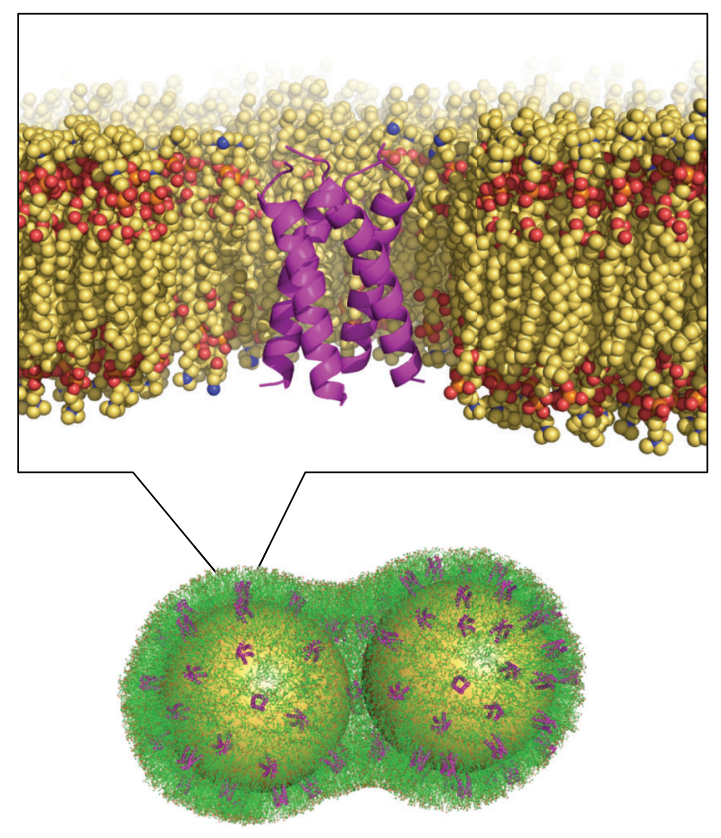

(a)

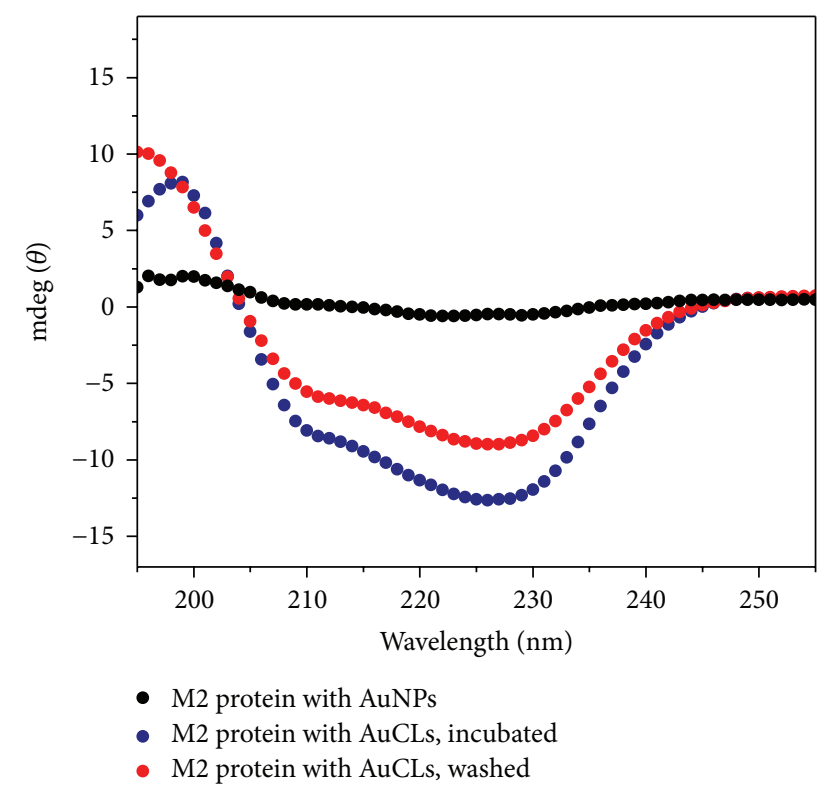

(b)

FIGURE 4: Secondary structure analysis of incorporated M2 proton channels in the lipid layers of AuCLs. (a) Illustration of an M2 protein, having a folded structure, in a lipid bilayer environment. The M2 protein has an inherent $\alpha$-helical conformation in cellular membrane environments. (b) Circular dichroism (CD) spectra of M2 proteins with citrate-capped AuNPs (black), incubated with AuCLs (blue), or after eliminating excess M2 protein in supernatant (red). Strong negative peaks appear at wavelengths of $208 \mathrm{~nm}$ and $222 \mathrm{~nm}$, which represent an $\alpha$-helix conformation.

removed nor exchanged with cellular lipids, which would result in fluorescence appearing on the peripheral cellular membrane. The mechanism of internalization is unknown 


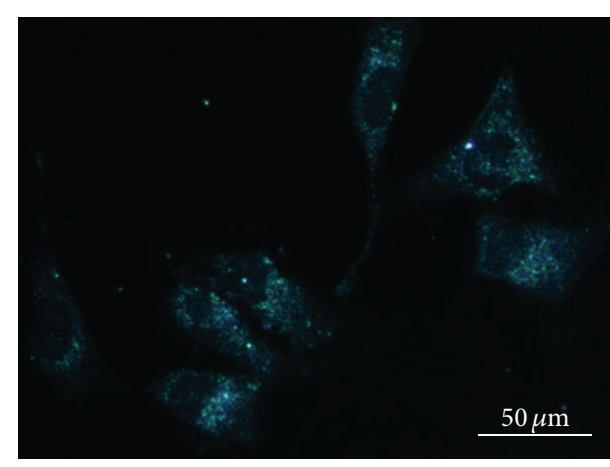

(a)

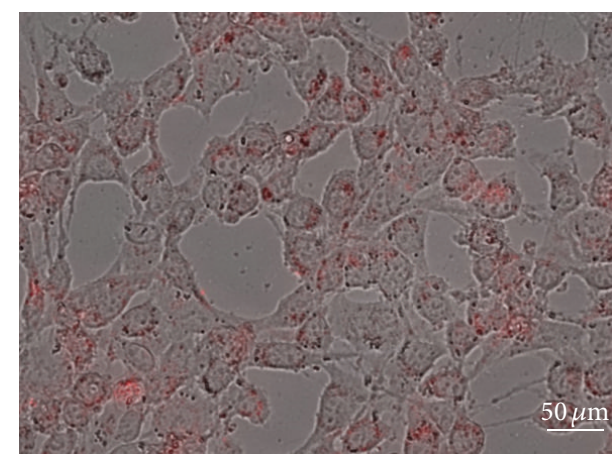

(b)

Figure 5: In vitro SH-SY5Y cellular uptake studies of AuCLs. (a) Dark field image and (b) confocal fluorescence image of SH-SY5Y neuroblastoma cells incubated with fluorescence-tagged AuCLs. $5 \mathrm{nM}$ of AuCLs was directly added to cell media and incubated for 1 hour. Images were taken after washing cells with fresh cell media.

at this stage. AuCLs can enter cells either by direct internalization [31] or through the mechanism of endocytosis [32]. If AuCLs entered the cell membrane through endocytosis, it would have the sequential addition of a lipid layer [33]. Even though further study of the internalization process is necessary, the microscopic data confirms the stability of lipid assemblies on AuNPs and suggests the possibility of developing AuCLs as a drug cargo.

\section{Conclusions}

We have demonstrated controlled clustering of AuNPs using cellular membrane mimicking phospholipid bilayers. Three different kinds of phospholipids, POPC, POPS, and POPE, were used, and the modulation of their concentration ratio successfully encapsulated AuNPs with an average size of $100 \mathrm{~nm}$ that were capable of self-assembling into dimers, trimers, and tetramers. High-resolution TEM images also showed AuNPs are enveloped by lipid layers in the oligomeric state, and the stability of these states lasted at least for 24 hours. In addition, the helical folding of the M2 proton channel, when it was incubated with AuCLs, was examined by CD spectrometer, and the results showed that M2 proteins form their inherent $\alpha$-helical structures in the presence of AuCLs. The results confirmed the formation of lipid bilayers on the nanoparticles and proved the possibility of functionalizing the surface of gold clusters with various, specific cell-targeting membrane proteins. Fluorescence-tagged AuCLs were also demonstrated to be capable of cellular entrance. In spite of the exposure to the cellular membrane, AuCLs retained their lipid layers while being internalized into cells. Our approach of clustering nanoparticles can be further developed to design nanoclusters with desired sizes. With the advantage of enhanced biocompatibility due to enveloping the surface of AuNPs with cell-like membranes, these AuCLs can also be used as imaging agents with enhanced spectroscopic sensitivity.

\section{Competing Interests}

The authors declare that there are no competing interests regarding the publication of this paper.

\section{Acknowledgments}

This work was supported by the National Research Foundation of Korea (NRF) under Grant NRF2015R1A2A2A04002453 and the Basic Science Research Program through the National Research Foundation of Korea (NRF) funded by the Ministry of Education (NRF2014R1A1A2055647 and NRF-2015M3C1A3002152).

\section{References}

[1] P. Pericleous, M. Gazouli, A. Lyberopoulou, S. Rizos, N. Nikiteas, and E. P. Efstathopoulos, "Quantum dots hold promise for early cancer imaging and detection," International Journal of Cancer, vol. 131, no. 3, pp. 519-528, 2012.

[2] P. K. Jain, X. Huang, I. H. El-Sayed, and M. A. El-Sayed, "Noble metals on the nanoscale: optical and photothermal properties and some applications in imaging, sensing, biology, and medicine," Accounts of Chemical Research, vol. 41, no. 12, pp. 1578-1586, 2008.

[3] D. Pissuwan, S. M. Valenzuela, and M. B. Cortie, "Therapeutic possibilities of plasmonically heated gold nanoparticles," Trends in Biotechnology, vol. 24, no. 2, pp. 62-67, 2006.

[4] K. Saha, S. S. Agasti, C. Kim, X. Li, and V. M. Rotello, "Gold nanoparticles in chemical and biological sensing," Chemical Reviews, vol. 112, no. 5, pp. 2739-2779, 2012.

[5] C. J. Murphy, A. M. Gole, J. W. Stone et al., "Gold nanoparticles in biology: beyond toxicity to cellular imaging," Accounts of Chemical Research, vol. 41, no. 12, pp. 1721-1730, 2008.

[6] S. Kang, S. H. Bhang, S. Hwang et al., "Mesenchymal stem cells aggregate and deliver gold nanoparticles to tumors for photothermal therapy," ACS Nano, vol. 9, no. 10, pp. 9678-9690, 2015. 
[7] L. R. Hirsch, R. J. Stafford, J. A. Bankson et al., "Nanoshellmediated near-infrared thermal therapy of tumors under magnetic resonance guidance," Proceedings of the National Academy of Sciences of the United States of America, vol. 100, no. 23, pp. 13549-13554, 2003.

[8] X. Qian, X.-H. Peng, D. O. Ansari et al., "In vivo tumor targeting and spectroscopic detection with surface-enhanced Raman nanoparticle tags," Nature Biotechnology, vol. 26, no. 1, pp. 83-90, 2008.

[9] D. Paria, K. Roy, H. J. Singh et al., "Ultrahigh field enhancement and photoresponse in atomically separated arrays of plasmonic dimers," Advanced Materials, vol. 27, no. 10, pp. 1751-1758, 2015.

[10] B. Khlebtsov, V. Zharov, A. Melnikov, V. Tuchin, and N. Khlebtsov, "Optical amplification of photothermal therapy with gold nanoparticles and nanoclusters," Nanotechnology, vol. 17, no. 20 , p. $5167,2006$.

[11] U. S. Dinish, G. Balasundaram, Y.-T. Chang, and M. Olivo, "Actively targeted in vivo multiplex detection of intrinsic cancer biomarkers using biocompatible SERS nanotags," Scientific Reports, vol. 4, article 4075, 2014.

[12] L.-J. Xu, C. Zong, X.-S. Zheng, P. Hu, J.-M. Feng, and B. Ren, "Label-free detection of native proteins by surface-enhanced Raman spectroscopy using iodide-modified nanoparticles," Analytical Chemistry, vol. 86, no. 4, pp. 2238-2245, 2014.

[13] E. Sackmann, "Supported membranes: scientific and practical applications," Science, vol. 271, no. 5245, pp. 43-48, 1996.

[14] A. Kloboucek, A. Behrisch, J. Faix, and E. Sackmann, "Adhesion-induced receptor segregation and adhesion plaque formation: a model membrane study," Biophysical Journal, vol. 77, no. 4, pp. 2311-2328, 1999.

[15] M. Tanaka and E. Sackmann, "Polymer-supported membranes as models of the cell surface," Nature, vol. 437, no. 7059, pp. 656663, 2005.

[16] S. P. Moura and A. M. Carmona-Ribeiro, "Biomimetic particles: optimization of phospholipid bilayer coverage on silica and colloid stabilization," Langmuir, vol. 21, no. 22, pp. 10160-10164, 2005.

[17] E. E. Ross, S.-W. Mok, and S. R. Bugni, "Assembly of lipid bilayers on silica and modified silica colloids by reconstitution of dried lipid films," Langmuir, vol. 27, no. 14, pp. 8634-8644, 2011.

[18] B. Dubertret, P. Skourides, D. J. Norris, V. Noireaux, A. H. Brivanlou, and A. Libchaber, "In vivo imaging of quantum dots encapsulated in phospholipid micelles," Science, vol. 298, no. 5599, pp. 1759-1762, 2002.

[19] L.-S. Wang, L.-C. Wu, S.-Y. Lu et al., "Biofunctionalized phospholipid-capped mesoporous silica nanoshuttles for targeted drug delivery: improved water suspensibility and decreased nonspecific protein binding," ACS Nano, vol. 4, no. 8, pp. 4371-4379, 2010.

[20] D. B. Chithrani, M. Dunne, J. Stewart, C. Allen, and D. A. Jaffray, "Cellular uptake and transport of gold nanoparticles incorporated in a liposomal carrier," Nanomedicine: Nanotechnology, Biology, and Medicine, vol. 6, no. 1, pp. e161-e169, 2010.

[21] N. Zhang, H. Chen, A.-Y. Liu et al., "Gold conjugate-based liposomes with hybrid cluster bomb structure for liver cancer therapy," Biomaterials, vol. 74, pp. 280-291, 2016.

[22] M. Wang, K. Alberti, S. Sun, C. L. Arellano, and Q. Xu, "Combinatorially designed lipid-like nanoparticles for intracellular delivery of cytotoxic protein for cancer therapy," Angewandte Chemie-International Edition, vol. 53, no. 11, pp. 2893-2898, 2014.
[23] A. L. Stouffer, R. Acharya, D. Salom et al., "Structural basis for the function and inhibition of an influenza virus proton channel," Nature, vol. 451, no. 7178, pp. 596-599, 2008.

[24] J. A. Yang and C. J. Murphy, "Evidence for patchy lipid layers on gold nanoparticle surfaces," Langmuir, vol. 28, no. 12, pp. 54045416, 2012.

[25] M. Antonietti and S. Förster, "Vesicles and liposomes: a selfassembly principle beyond lipids," Advanced Materials, vol. 15, no. 16, pp. 1323-1333, 2003.

[26] I. R. Cooke and M. Deserno, "Coupling between lipid shape and membrane curvature," Biophysical Journal, vol. 91, no. 2, pp. 487-495, 2006.

[27] J. M. Romo-Herrera, R. A. Alvarez-Puebla, and L. M. LizMarzán, "Controlled assembly of plasmonic colloidal nanoparticle clusters," Nanoscale, vol. 3, no. 4, pp. 1304-1315, 2011.

[28] H. T. McMahon and J. L. Gallop, "Membrane curvature and mechanisms of dynamic cell membrane remodelling," Nature, vol. 438, no. 7068, pp. 590-596, 2005.

[29] K. C. Duff, S. M. Kelly, N. C. Price, and J. P. Bradshaw, “The secondary structure of influenza A M2 transmembrane domain A circular dichroism study," FEBS Letters, vol. 311, no. 3, pp. 256258, 1992.

[30] F. A. Kovacs and T. A. Cross, "Transmembrane four-helix bundle of influenza A M2 protein channel: Structural implications from helix tilt and orientation," Biophysical Journal, vol. 73, no. 5, pp. 2511-2517, 1997.

[31] P. Nativo, I. A. Prior, and M. Brust, "Uptake and intracellular fate of surface-modified gold nanoparticles," ACS Nano, vol. 2, no. 8, pp. 1639-1644, 2008.

[32] B. D. Chithrani and W. C. W. Chan, "Elucidating the mechanism of cellular uptake and removal of protein-coated gold nanoparticles of different sizes and shapes," Nano Letters, vol. 7, no. 6, pp. 1542-1550, 2007.

[33] J. Sun, L. Zhang, J. Wang et al., "Tunable rigidity of (polymeric core)-(lipid shell) nanoparticles for regulated cellular uptake," Advanced Materials, vol. 27, no. 8, pp. 1402-1407, 2015. 

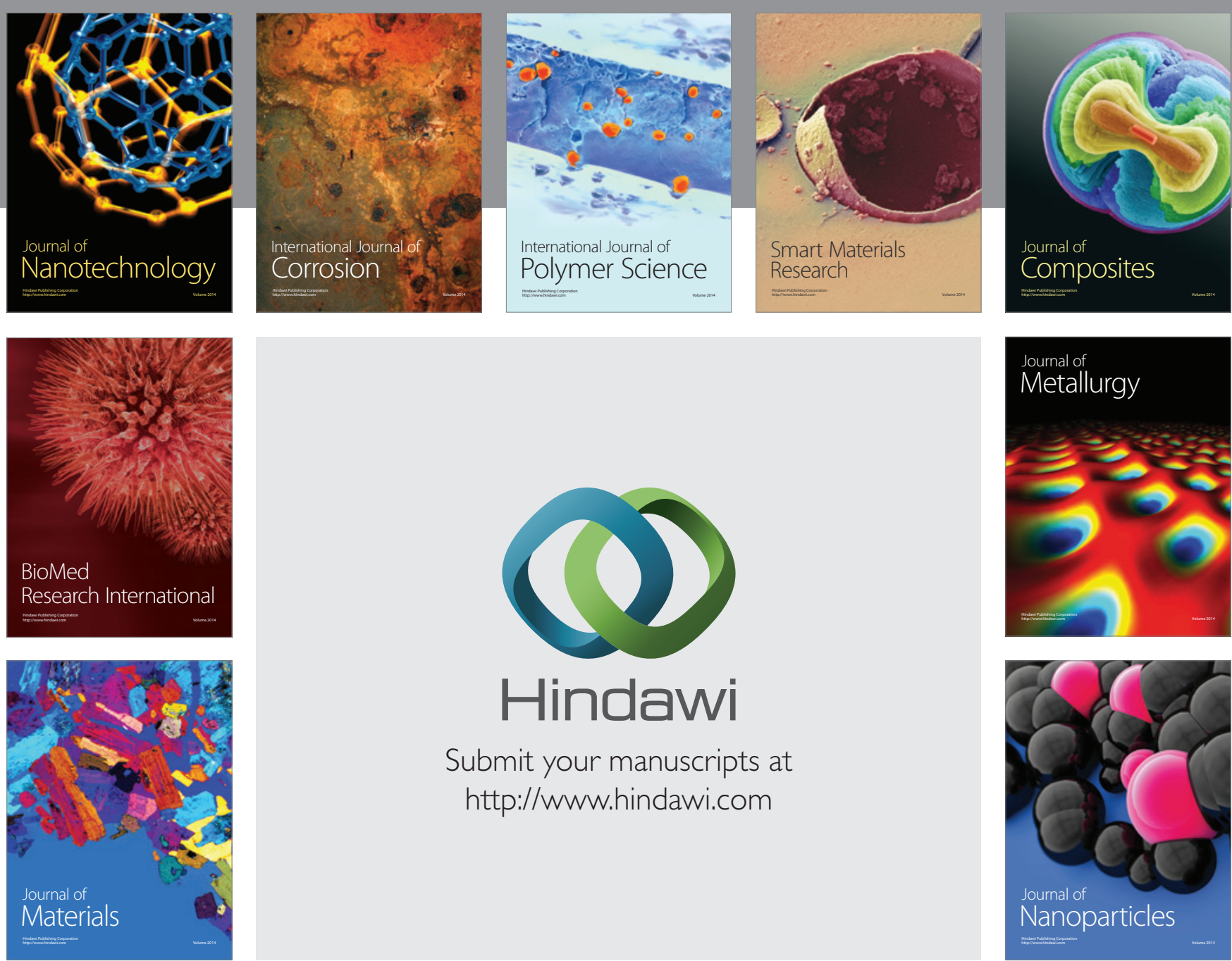

\section{Hindawi}

Submit your manuscripts at

http://www.hindawi.com

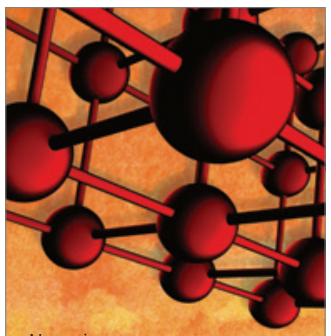

Materials Science and Engineering
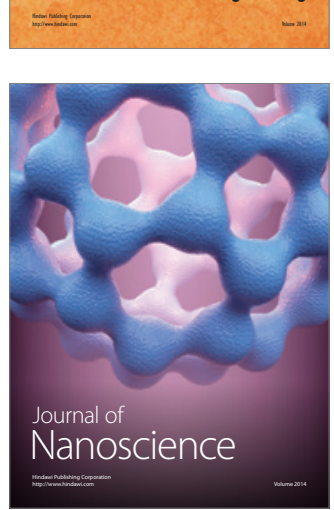
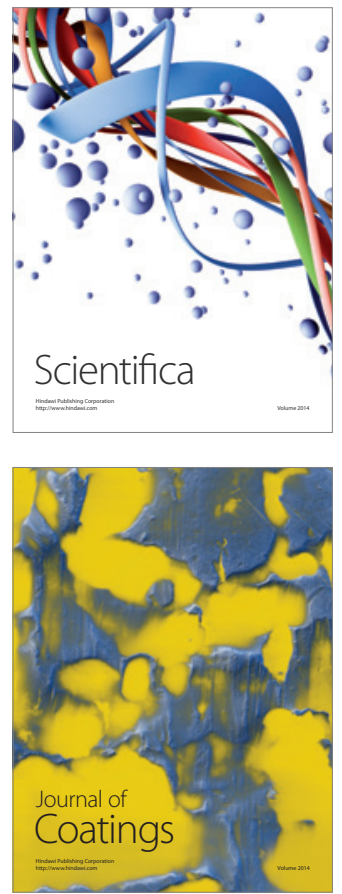
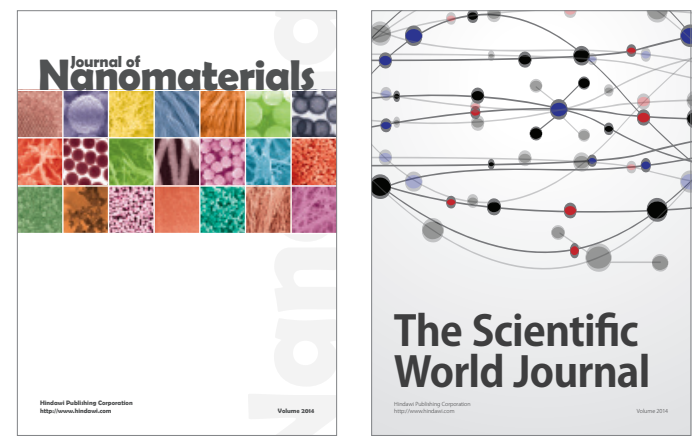

The Scientific World Journal
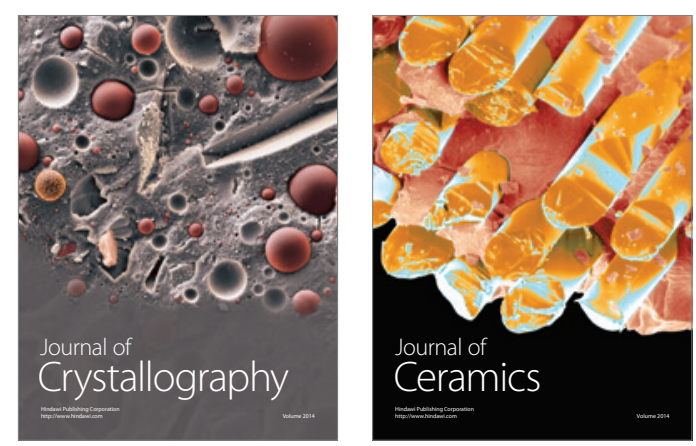
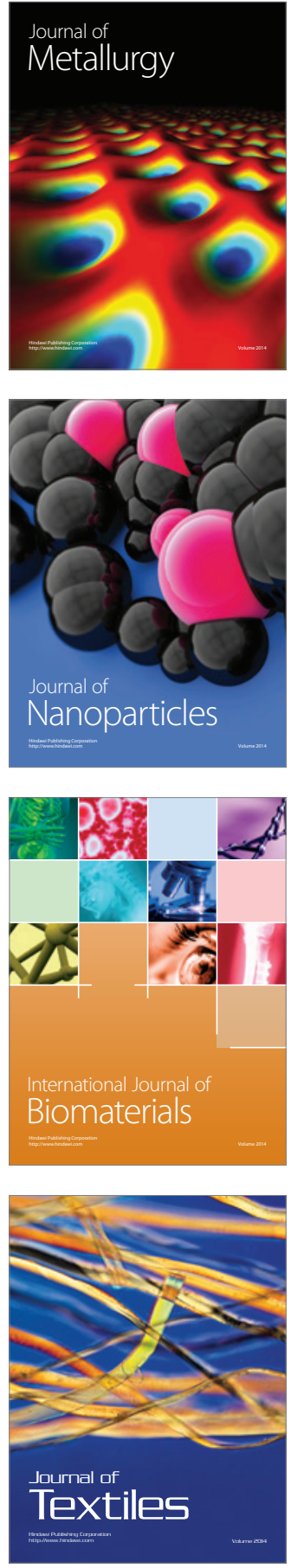